\title{
Fission fragments observables measured at the LOHENGRIN spectrometer
}

\author{
S. Julien-Laferrière ${ }^{1,2}, L$. Thombansen ${ }^{2}, G$. Kessedjian ${ }^{2}, A$. Chebboubi $^{1}, O$. Serot ${ }^{1}, C$. Sage ${ }^{2, *}, O$. Méplan $^{2}, M$.

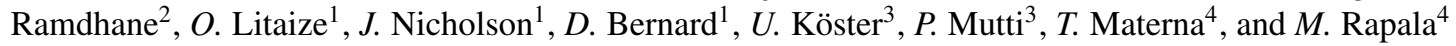 \\ ${ }^{1}$ CEA, DEN, DER, SPRC, LEPh, Cadarache center, F-13108 Saint-Paul-lez-Durance, France \\ ${ }^{2}$ LPSC, Université Grenoble-Alpes, CNRS/IN2P3, F-38026 Grenoble Cedex, France \\ ${ }^{3}$ Institut Laue-Langevin, F-38042 Grenoble Cedex 9, France \\ ${ }^{4}$ CEA, DSM, IRFU, SPhN, LEARN, Saclay center, F-91191 Gif-sur-Yvette, France
}

\begin{abstract}
Nuclear fission yields are key data for reactor studies, such as spent fuel inventory or decay heat, and for understanding fission process. Despite a significant effort allocated to measure fission yields during the last decades, the recent evaluated libraries still need improvements in particular in the reduction of the uncertainties. Moreover, some discrepancies between these libraries must be explained.

Additional measurements provide complementary information and estimations of experimental correlations, and new kinds of measurements enable to test the models used during the nuclear data evaluation process. A common effort by the CEA, the LPSC and the ILL aims at tackling these issues by providing precise measurements of isotopic and isobaric fission yields with the related variance-covariance matrices. Additionally, the experimental program involves a large range of observables requested by the evaluations, such as kinetic energy dependency of isotopic yields and odd-even effect in order to test the sharing of total excitation energy and the spin generation mechanism. Another example is the complete range of isotopic distribution per mass that allows the determination of the charge polarization, which has to be consistent for complementary masses (pre-neutron emission). For instance, this information is the key observable for the evaluation of isotopic yields. Finally, ionic charge distributions are indirect measurements of nanosecond isomeric ratios as a probe of the nuclear de-excitation path in the $\left(E^{*}, J, \pi\right)$ representation.

Measurements for thermal neutron induced fission of ${ }^{241} \mathrm{Pu}$ have been carried out at the ILL in Grenoble, using the LOHENGRIN mass spectrometer. Methods, results and comparison to models calculations will be presented corresponding to a status on fission fragments observables reachable with this facility.
\end{abstract}

\section{Introduction}

An accurate knowledge of fission data in the actinide region is important for studies of innovative nuclear reactor concepts. Fission yield measurements supply experimental data to put constraints on fission models and improve their predictive power. In the framework of nuclear data evaluation, these models are indeed necessary to increase the consistency and the precision of the libraries. Despite a real effort on fission yields measurements, current evaluated data still need some improvements on different aspects, such as for instance the uncertainties reduction and the estimation of covariance matrices. A special focus on the heavy and symmetry mass regions is important, since it is where the discrepancies between models (or evaluations) and the few experimental data are mainly observed.

A collaboration between the CEA, the LPSC and the Institute Laue Langevin (ILL) is involved in an experimental program using thermal neutrons of the ILL and the LOHENGRIN spectrometer to study the fission process. We developed different methodologies to obtain absolute isobaric and isotopic yields with the estimation of the covariance matrices associated to the measurements. Besides,

*e-mail: sage@1psc.in2p3.fr the measurement of different observables combined with a comparision with simulation codes such as FIFRELIN [1] enable to get insight data to better understand the fission process. Isomeric ratios can give an indirect information on the fragments spin ditribution, and their kinetic energy dependency enlights on the validity of the models in use. An exhaustive set of isotopic yields per mass enables the charge polarisation estimation, which has to be consistent between complementary masses. A final example of such indirect data measured by our collaboration is the extraction of nanosecond isomeric ratios determined from the ionic charge distributions per isotope.

\section{The LOHENGRIN spectrometer}

The LOHENGRIN mass spectrometer [2] is a nuclear physics instrument from the ILL research reactor facility which allows to study fragment distributions from thermal neutron induced fission with a very high mass resolution $(\Delta A / A \approx 1 / 400)$. A fissile actinide target is placed close to the reactor core, in a thermal neutron flux reaching $5 \times 10^{14}$ neutron. $\mathrm{cm}^{-2} \cdot \mathrm{s}^{-1}$.

Fission fragments emerge from the target with an ionic charge distributed around an average ionic charge state of 
about 20 to 23. Those fragments that are emitted along the beam tube axis undergo a horizontal deflection in a magnetic field, directly followed by a vertical deflection in an electric field. These combined fields separate ions according to their $A / q$ and $E_{k} / q$ ratios, with $A, q$ and $E_{k}$ the mass, ionic charge state and kinetic energy of the ions respectively. These ratios can be achieved with different triplets $\left(A, E_{k}, q\right)$ leading to a possible degeneracy.

At the spectrometer exit, different detection systems can be installed, such as a dual anode Frisch grid ionisation chamber for mass yield measurements, or two clovers of four high purity Germanium crystals that are used with an additional magnet whose aim is to focus the ion beam. A schematic view of the spectrometer is shown in Fig. 1.

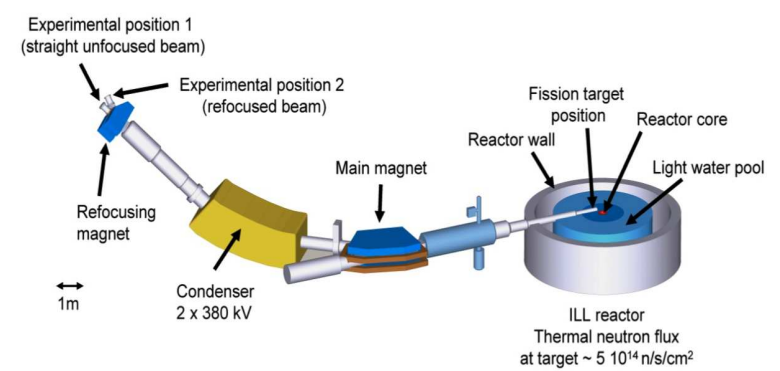

Figure 1. Schematic overview of the LOHENGRIN spectrometer at ILL.

\section{Mass and isotopic yields measurements of the ${ }^{241} \mathrm{Pu}\left(\mathbf{n}_{t h}, \mathbf{f}\right)$ reaction}

The first goal of our collaboration measurement campaign consists in the precise measurements of mass, isotopic and isomeric yields, with a control of the systematic effects and the determination of the covariance matrices associated to the analyses. For these observables, their dependency with fission fragment kinetic energy increases significantly the retrieved information on the fission process.

\subsection{Mass yields}

Isobaric yields are obtained from experimental position 1 (see Fig. 1) after an integration over the kinetic energy and the ionic charge distributions of the count rates measured with the ionisation chamber. A new measurement method and consequent analysis path have been developed and are detailed in Ref. [3-6]. Among the special features of this method are the self-normalisation of our data and the calculation of the experimental covariance matrices. Provided that all the heavy mass rates are measured, it is possible to self-normalise the data by defining to $100 \%$ the sum of the whole heavy peak yields. As a consequence, these new measurements are independent from another experiment or assessment and may be compared directly with the existing data and evaluations.

The results for ${ }^{241} \mathrm{Pu}\left(\mathrm{n}_{t h}, \mathrm{f}\right)$ are shown in Fig. 2, where they are compared to the JEFF-3.3 [7] and ENDF/B-VII.1 [8] libraries. The whole heavy peak and an important part of the light one were measured. Our results are slightly higher than the libraries for the light mass region, and a structure around mass 140 is observed in the heavy region. Our experimental uncertainties are around 5\% on average and below the ones indicated in the two libraries.

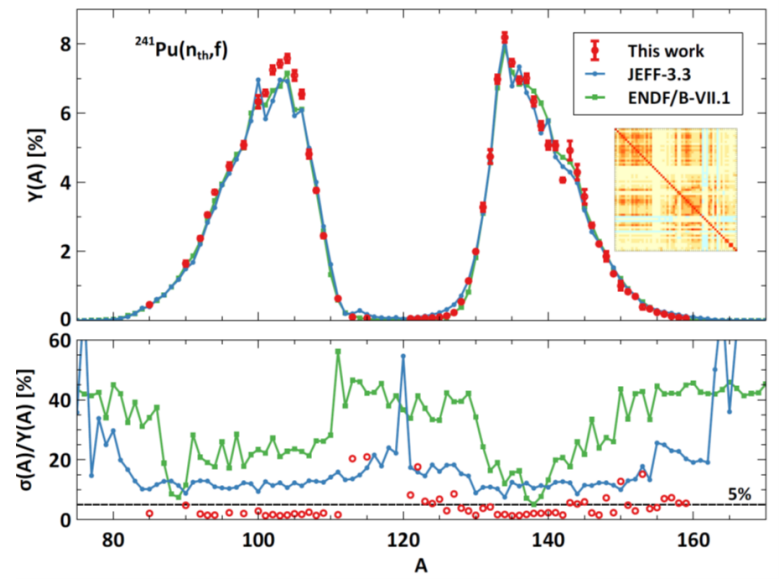

Figure 2. Mass yields for the ${ }^{241} \mathrm{Pu}\left(\mathrm{n}_{t h}, \mathrm{f}\right)$ reaction and their relative uncertainties.

\subsection{Isotopic yields}

Isotopic and long-lived isomeric (few $\mu$ s to ms) fission yields are measured by gamma spectrometry. Experimental position 2 (see Fig. 1) is now used.The ion beam is deposited on a moving tape inside a vacuum chamber and a cumulative measurement with duration of about $30 \mathrm{~min}$ per point over the ionic charge distribution is achieved. The tape moves at the end of the measurement to clean the environment and start a new measurement. After corrections of the Bateman equations and the estimation of the contribution of the isotopes from the tape only, we obtain the isotopic distributions per mass. As for the mass yields, a particular effort is made on the determination of the systematic uncertainties and the covariance associated to the measurement process.

Fig. 3 shows a scheme summarizing the measurement procedure and the isotopic yields obtained for the chains 137 and 139 are shown in Fig. 4, along with the covariance matrices. It is important to note that the uncertainties are dominated by the nuclear structure data. Thus current yields measurements can be improved by increasing the nuclear structure knowledge. Fig. 5 helps to understand the construction of the experimental covariance matrix at the main steps of the analysis as illustration of the uncertainty propagation effects.

\section{Indirect data measurements}

Besides isobaric and isotopic yields, other fission observables are achievable with the LOHENGRIN spectrometer and give important complementary information for the study of the fission process. The kinetic energy dependency of the isotopic and isomeric yields has been already 


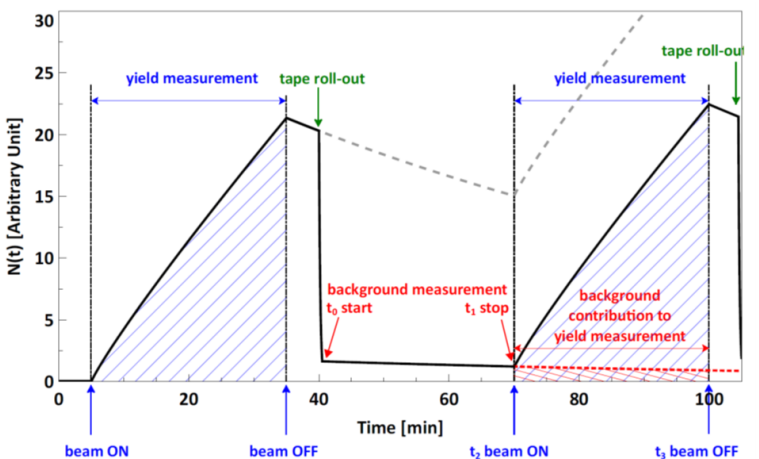

Figure 3. Evolution of a typical isotopic yields measurement procedure.
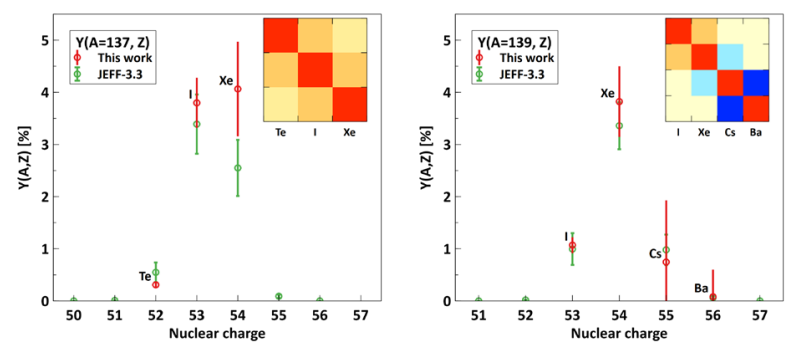

Figure 4. Isotopic yields of the ${ }^{241} \mathrm{Pu}\left(\mathrm{n}_{t h}, \mathrm{f}\right)$ reaction for masses 137 and 139, along with the experimental covariance matrices
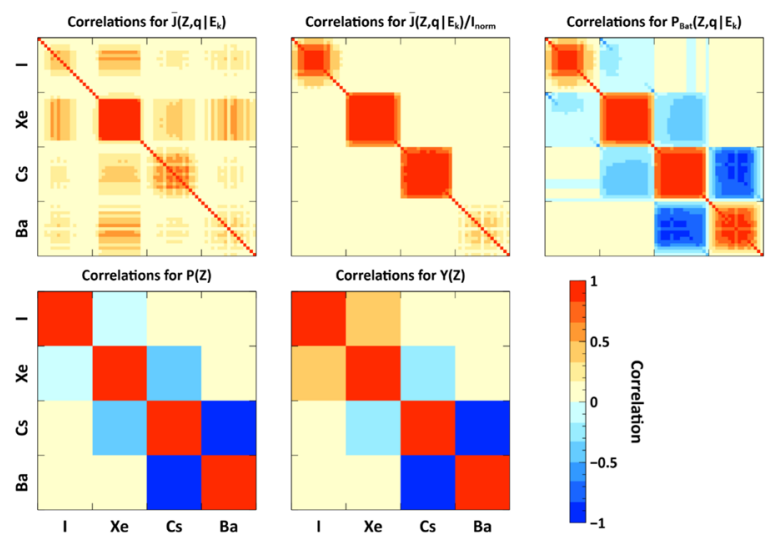

Figure 5. The correlation matrix for mass 139 at different steps of the analysis. $1^{\text {st }}$ step: average on the different gamma rays. $2^{\text {nd }}$ step: Division by $I^{\text {norm }}$. $3^{\text {rd }}$ step: Independent production rate calculation. $4^{\text {th }}$ step: sum over the ionic charges. $5^{\text {th }}$ step: absolute yields after self normalisation.

discussed in ref. $[9,10]$. This paper will focus on the description of the charge polarisation and the estimation of nanosecond isomeric ratios.

\subsection{Nuclear charge polarisation}

The charge polarisation can be extracted from the combination of the isotopic and isobaric yields measurements. It is defined as the difference between the measured mean nuclear charge and the fragment nuclear charge in the Unchanged Charge Density (UCD) hypothesis.

Fig. 6 shows the measured charge polarisation for the concerned masses in the heavy peak region, compared with the JEFF-3.1.1 library and previous experimental data from Schillebeeckx et al. [11]. We observe a good agreement for the mass 130 and around mass 140, but a strong structure appears for the masses 132, 136 and 138. Complementary measurements on the neighbouring masses are planned by the collaboration to better understand this phenomenon.

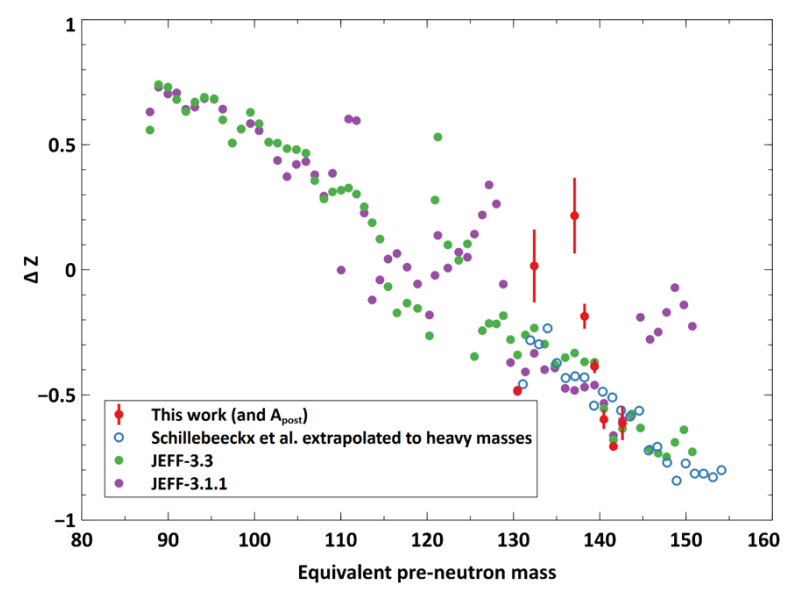

Figure 6. Charge polarisation measured in the heavy peak region as a function of the pre-neutron mass, compared with the JEFF3.1.1 library and previous experimental data from Schillebeeckx et al. [11].

\subsection{Nanosecond converted isomeric ratios}

Indirect measurements of nanosecond IR's can be determined from the ionic charge distributions [12, 13]. The method consists in the deconvolution of the ionic charge distribution per isotope obtained by gamma spectrometry after correction from Bateman equations. The converted isomeric ratio (CIR) is defined as the converted isomer population over the total ionic population (converted and unconverted).

$$
C I R=\frac{N\left(A, Z, m \rightarrow e^{-}\right)}{N(A, Z, G S)+N(A, Z, m \rightarrow \gamma)+N\left(A, Z, m \rightarrow e^{-}\right)}
$$

According to the statistical models from H. Betz [14], we assume that the ionic charge distribution associated to the unconverted population follows a Gaussian distribution due to the charge equilibrium in the cover of the target (a Nickel foil in this work). A deviation from this Gaussian distribution indicates a charge modification due to the conversion from ps and ns isomers to groundstate (see Fig. 7). In most of the cases the deconvolution is achieved using two Gaussian distributions and a Monte Carlo simulation to deduce the CIR in order to consider the covariance terms between the Gaussian integrals. CIR measurements for ${ }^{241} \mathrm{Pu}\left(\mathrm{n}_{t h}, \mathrm{f}\right)$ and ${ }^{233} \mathrm{U}\left(\mathrm{n}_{t h}, \mathrm{f}\right)$ are shown in Fig. 8 and compared to FIFRELIN calculations for ${ }^{241} \mathrm{Pu}\left(\mathrm{n}_{t h}, \mathrm{f}\right)$. We note 


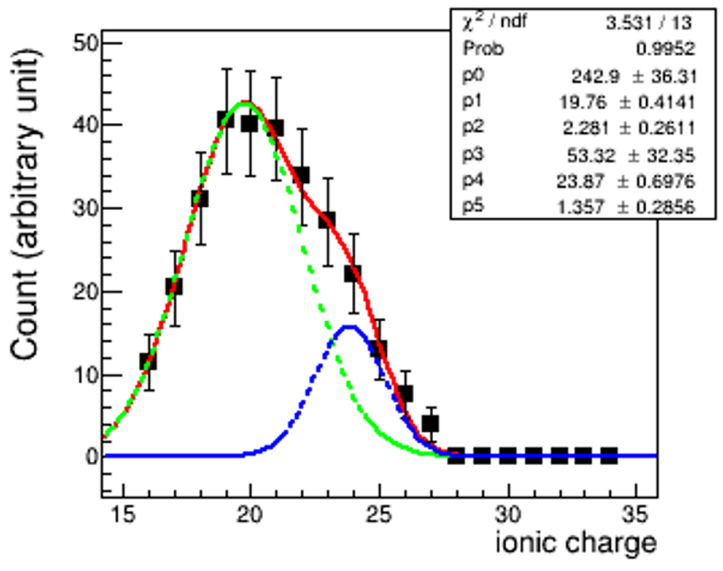

Figure 7. Ionic charge distribution de-convoluted using the Gaussian assumption for the unconverted state according to the Betz model [14]. The blue curve corresponds to the ns isomer contribution.

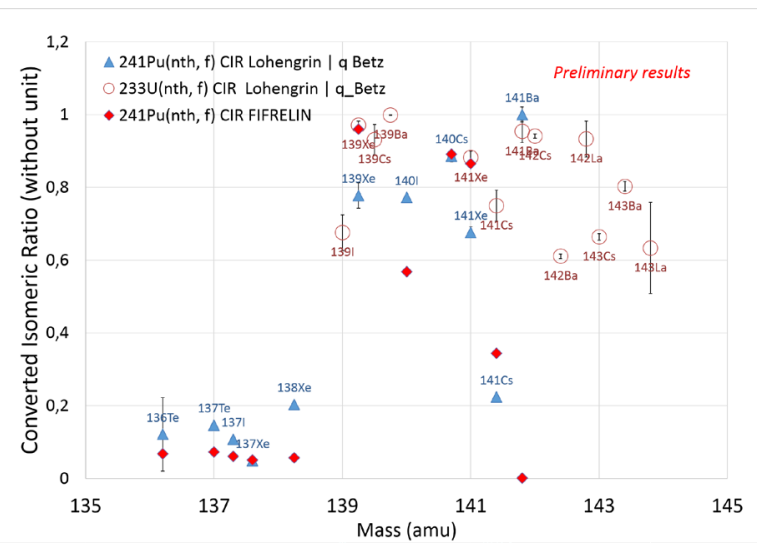

Figure 8. Converted isomeric ratios as a function of the mass induced by the fission of ${ }^{241} P u\left(n_{t h}, \mathrm{f}\right)$ and ${ }^{233} U\left(n_{t h}, \mathrm{f}\right)$ in comparison with FIFRELIN calculations.

a good tendency even if some differences have to be explored in details given the assumption used in the analysis or the models considered for the decay cascade calculations. For some nuclei, many isomers and bands have to be taken into account at the limit of the knowledge of the nuclear structure. Then it corresponds to integral measurements used to test the overal decay cascade.

\section{Conclusion and perspectives}

An experimental program dedicated to precise absolute measurements of isobaric, isotopic and isomeric yields is ongoing using the LOHENGRIN mass spectrometer at ILL. Recent results concern the ${ }^{241} \mathrm{Pu}\left(\mathrm{n}_{t h}, \mathrm{f}\right)$ reaction, where a dedicated analysis method with a control of the systematic uncertainties and computation of the covariance matrices was achieved. Interesting indirect data are also measured and dedicated to test the phenomenological models and the assumptions used for the evaluations, through a comparison with calculations using the FIFRELIN code developped at CEA Cadarache. In this frame, nuclear charge polarisation and nanosecond CIR are the main examples of such investigations. Our collaboration plans to continue this measurement program for different fissioning systems, as new measurements and validated models are central in order to progress in the evaluation topic.

\section{Acknowledgements}

This work was supported by CEA, IN2P3 and "le défi NEEDS". The authors are grateful for the support of the ILL and all the staff involved from CEA Cadarache and LPSC.

\section{References}

[1] O. Litaize and O. Serot, Eur. Phys. J . A 51, 177 (2015)

[2] P. Armbruster et al., Nucl. Instrum. Methods 139213 (1976)

[3] F. Martin et al., Proc. 2nd ANIMMA Conference, June 2011, Ghent (Belgium).

[4] F. Martin et al., Nuclear Data Sheets 119, 328-330 (2014)

[5] S. Julien-Laferrière et al., EPJ Nucl. Sci. Technol. 4, 25 (2018)

[6] Y. Gupta et al., Phys. Rev. C 96, 014608 (2017)

[7] The JEFF-3.1.1 nuclear data library. OECD, NEA, JEFF Report 21, ISBN 92-64-02314-3 (2006).

[8] M. B. Chadwick et al., Nucl. Data Sheets 107, 2931 (2006)

[9] S. Julien-Laferrière et al., EPJ Web of Conferences 211, 04004 (2019)

[10] A. Chebboubi et al., Phys. Lett. B 775, 190-195 (2017)

[11] P. Schillebeeckx et al., Nucl. Phys. A 580, 15-32 (1994)

[12] T. Rzaca-Urban, J. Genevey, T. Materna et al., Phys. Rev. C 80, 064317 (2009)

[13] A. Chebboubi et al., EPJ Web of Conferences 146, 04021 (2017)

[14] H. D. Betz, Rev. Mod. Phys. 44, 465-539 (1972)

[15] R. Capote et al., Nucl. Data Sheets 110, 3107 (2009) 